\title{
MOBILIZAÇÃO DAS HABILIDADES E ESTRATÉGIAS METACOGNITIVAS POR MEIO DOS JOGOS DIGITAIS
}

MOBILIZING METACOGNITIVE SKILLS AND STRATEGIES THROUGH DIGITAL GAMESTÍTULO

\section{MOVILIZACIÓN DE HABILIDADES Y ESTRATEGIAS METACOGNITIVAS A TRAVÉS DE LOS JUEGOS DIGITALES}

\author{
Valdick Barbosa de Sales Junior ${ }^{1}$ \\ Fernando Silvio Cavalcante Pimentel ${ }^{2}$
}

\section{RESUMO}

A metacognição se refere ao conhecimento, monitoramento e controle das habilidades cognitivas de uma pessoa antes, durante e após o início da aprendizagem. A literatura mostra que a obtenção do conhecimento pelo aluno que tem uma relação significativa com o desempenho escolar, mas nem todas as estratégias de aprendizagem utilizadas têm o mesmo resultado. Além disso, a tecnologia educacional, especialmente com os jogos digitais, se desenvolveu rapidamente na última década e teve um impacto profundo em todos os níveis de educação. Essa Revisão Sistemática de Literatura (RSL), propõe o estudo em várias bases de conhecimento, sobre a mobilização da metacognição nos jogos digitais para responder à pergunta: os alunos que utilizam os jogos digitais em seu aprendizado, fazem uso da consciência, habilidades e estratégias metacognitivas? Fazendo uso da Ferramenta StArt (State of the Art through Systematic Review), 770 estudos foram extraídos de diversas bases de dados de

Submetido em: 12/07/2021 - Aceito em: 30/08/2021 - Publicado em: 13/10/2021

1 Mestre em Ciência da Computação pela UFAL e doutorando em Educação, PPGE- UFAL. Graduado em Ciência da Computação pela UFPE, especialista em Redes e Bancos de Dados pela Universidade Federal de Campina Grande (UFCG).,. É instrutor da Academia de Polícia Militar Senador Arnon De Mello, nos cursos de CFO, CAO e CSP. Foi criador do portal Gazetaweb.com, foi criador e editor do suplemento Gazeta Digital que circulou às quartas-feiras na Gazeta de Alagoas. Atualmente é consultor de tecnologia do quadro Universo Digital na TV Gazeta de Alagoas, afiliada Rede Globo, no Bom Dia Alagoas, foi diretor de Planejamento e Controle da Organização Arnon de Mello durante 21 anos. Consultor de Tecnologias Digitais há mais de 20 anos. É professor adjunto e Coordenador do Curso de Ciência da Computação da Faculdade da Cidade de Maceió - FACIMA, CEO da empresa AlPlace - Negócios Digitais.

2 Doutor e Mestre em Educação pela Universidade Federal de Alagoas, especialista em Tecnologias em Educação e Docência do Ensino Superior. Graduado em Pedagogia (Licenciatura). Foi professor da Educação Básica em escolas particulares, tutor no programa de formação continuada da Secretaria de Educação a Distância (MEC), professor tutor 1 na Universidade Tiradentes e professor no Governo do Estado de Alagoas. Tem experiência na área de Educação, com ênfase em Ensino-Aprendizagem, atuando principalmente nos seguintes temas: aprendizagem, jogos digitais na educação, gamificação, metodologias ativas, educação online, webquest, tutoria e avaliação. Foi Vice-coordenador da Coordenadoria Institucional de Educação a Distância e Coordenador Adjunto da UAB/UFAL. Integrante do banco de avaliadores institucionais do INEP, atualmente é líder do Grupo de Pesquisas Comunidades Virtuais - Ufal. É professor adjunto da Universidade Federal de Alagoas, onde exerceu a função de Coordenador do Programa de Pós-Graduação em Educação (PPGE). 
conhecimento e passaram pelos processos de identificação, triagem, seleção e qualidade para chegar ao número de 6 artigos que satisfizeram os critérios adotados. Como resultado, compreende-se que se trata de um estudo novo para a literatura. Nos dados, encontramos que $75 \%$ fazem uso das habilidades metacognitivas, $63 \%$ do conhecimento metacognitivo e $67 \%$ utilizam os processos cognitivos nos jogos.

PALAVRAS-CHAVE: Metacognição. Jogos digitais. Aprendizagem.

\section{ABSTRACT}

Metacognition refers to the knowledge, monitoring and control of a person's cognitive abilities before, during and after the start of learning. The literature shows that the student's attainment of knowledge that has a significant relationship with school performance, but not all learning strategies used have the same result. In addition, educational technology, especially with digital games, has developed rapidly in the last decade and has had a profound impact on all levels of education. This Systematic Literature Review (SLR), proposes the study in various knowledge bases, about the mobilization of metacognition in digital games to answer the question: do students who use digital games in their learning, make use of metacognitive awareness, skills and strategies? Making use of the StArt Tool (State of the Art through Systematic Review), 770 studies were extracted from various knowledge databases and went through the processes of identification, screening, selection and quality to arrive at the number of 6 articles that met the criteria adopted. As a result, it is understood that this is a new study for the literature. In the data, we found that $75 \%$ make use of metacognitive skills, $63 \%$ metacognitive knowledge and $67 \%$ use cognitive processes in games.

\section{KEYWORDS: Metacognition. Digital games. Learning. RESUMEN}

La metacognición se refiere al conocimiento, seguimiento y control de las habilidades cognitivas de una persona antes, durante y después del inicio del aprendizaje. La literatura muestra que la adquisición de conocimientos por parte del alumno tiene una relación significativa con el rendimiento escolar, pero no todas las estrategias de aprendizaje utilizadas tienen el mismo resultado. Además, la tecnología educativa, especialmente con los juegos digitales, se ha desarrollado rápidamente en la última década y ha tenido un profundo impacto en todos los niveles de la educación. Esta Revisión Sistemática de la Literatura (RSL), propone el estudio en diversas bases de conocimiento, sobre la movilización de la metacognición en los juegos digitales para responder a la pregunta: ¿los estudiantes que utilizan juegos digitales en su aprendizaje, hacen uso de la conciencia, habilidades y estrategias metacognitivas? Haciendo uso de la herramienta StArt (State of the Art through Systematic Review), se extrajeron 770 estudios de diversas bases de datos de conocimiento y pasaron por los procesos de identificación, cribado, selección y calidad hasta llegar al número de 6 artículos que cumplían los criterios adoptados. En consecuencia, se entiende que este es un estudio nuevo para la literatura. En los datos, encontramos que el $75 \%$ hace uso de habilidades metacognitivas, el $63 \%$ de conocimientos metacognitivos y el $67 \%$ de procesos cognitivos en los juegos.

PALABRAS CLAVE: Metacognición. Juegos digitales. Aprendizaje.

\section{INTRODUÇÃO}

No contexto da Cultura Digital, quando as tecnologias digitais estão cada vez mais presentes no dia a dia das instituições de ensino, os jogos digitais também estão presentes nesse contexto possibilitando uma série de benefícios para as práticas de ensino e aprendizagem (PRENSKY, 2003a). Essa realidade tem sido ampliada nos últimos meses, em decorrência da pandemia da Covid-19, já que estas tecnologias podem colaborar na tentativa de diminuir os prejuízos causados pelo afastamento e distanciamento social. 
Segundo Savi e Ulbricht (2008), conseguir desviar a atenção que os estudantes dão aos jogos para atividades educacionais não é tarefa simples. Por isso, tem aumentado o número de pesquisas que tentam encontrar formas de unir ensino e diversão com o desenvolvimento de jogos digitais (Pimentel et al, 2021). Por proporcionarem práticas educacionais atrativas e inovadoras, onde o aluno tem a chance de aprender de forma mais ativa, dinâmica e motivadora, os jogos digitais podem se tornar auxiliares no processo de seu aprendizado.

No entanto, para serem usados para fins educacionais, os jogos precisam ter objetivos de aprendizagem claros e ensinar o conteúdo das disciplinas aos alunos, ou promover o desenvolvimento de estratégias ou habilidades importantes para expandir as habilidades cognitivas e intelectuais dos alunos (GROS, 2003). Neste estágio, ressalta Flavell (1979, p. 907), surge a metacognição que consiste em dois componentes principais: conhecimento metacognitivo e a regulação metacognitiva.

O conhecimento metacognitivo refere-se ao conhecimento da cognição, ou seja, o conhecimento das habilidades e estratégias mais adequadas para os alunos e o conhecimento de como e quando usar essas habilidades e estratégias. O conhecimento da cognição refere-se a que os indivíduos sabem sobre sua própria cognição ou sobre cognição em geral; e a regulação da cognição inclui atividades metacognitivas que ajudam a controlar o pensamento ou a aprendizagem. A compreensão destes conceitos pode colaborar com uma proposta educacional em que os estudantes sejam mais proativos, como na aprendizagem baseada em jogos digitais (PRENSKY, 2003b; EGENFELDT-NIELSEN, 2010; VAN ECK, 2015).

Sobre a necessidade do indivíduo ter plena consciência dos objetivos que deseja alcançar, Zimmerman (2008) comenta a necessidade de conhecer as demandas e as exigências da tarefa; procurar realizar as ações; avaliar o nível de realização atingido e, num processo cíclico, alterar, se necessário, os procedimentos adotados quando não alcançar os resultados esperados.

Partindo desta relação entre os jogos digitais e a metacognição, descobrir quais os processos que permitem monitorar, regular e controlar a cognição com jogos digitais, nos remete ao seguinte problema: como as habilidades e estratégias metacognitivas desenvolvidas pelos estudantes que fazem uso de jogos digitais são mobilizadas para sua própria aprendizagem?

Este artigo apresenta uma Revisão Sistemática da Literatura (RSL), que teve como objetivo mapear os estudos realizados no contexto do desenvolvimento metacognitivo a partir da utilização dos jogos digitais no ensino superior. O artigo é subdividido em duas partes. Uma parte teórica, quando se apresentam os conceitos de metacognição e a compreensão do com jogos digitais na educação. 
A segunda parte apresenta a metodologia da investigação, indicando os trabalhos correlatos, o passo a passo da RSL a discussão e a análise dos dados encontrados após os procedimentos de coleta, triagem e categorização.

\section{METACOGNIÇÃO}

A metacognição refere-se a qualquer conhecimento e processo cognitivo relacionado à avaliação, monitoramento e ajuste da cognição (FLAVELL, 1979). Segundo (FUND, 2018) envolve a maneira como os alunos monitoram e orientam propositalmente seu aprendizado. Por exemplo, depois de determinar que uma estratégia cognitiva específica para a memorização provavelmente terá sucesso, o estudante então monitora se ela foi realmente bem-sucedida e, deliberadamente muda (ou não muda) seu método de memorização com base nas evidências. Por meio de estratégias metacognitivas, é possível compreender as estratégias utilizadas para monitorar ou controlar a cognição, como verificar se nossa técnica de memorização foi precisa ou escolher a estratégia cognitiva mais adequada para a tarefa realizada.

Para Dembo (2001), as estratégias cognitivas referem-se a comportamentos e pensamentos que influenciam o processo de aprendizagem, de maneira que a informação possa ser armazenada mais eficientemente, e as metacognitivas são os procedimentos que o indivíduo usa para planejar, monitorar e regular o seu próprio pensamento.

Além disso, aprendendo a aprender entrou em cena na medida em que foi questionado como os estudantes poderiam se tornar aprendizes independentes. Isto é, como poderiam se tornar responsáveis pela construção do conhecimento cognitivo, avançando sua aprendizagem por vontade própria. Para Van Velzen (2015), esta parecia ser uma questão essencial, particularmente porque o foco nas escolas é uma clara apreensão do assunto e dificilmente aprender a aprender.

Flavell (1979, p. 906) em sua pesquisa na Stanford University no final dos anos 1970 e 1980 foi quem primeiro apresentou um trabalho na área definido como o conhecimento e cognição sobre o fenômeno cognitivo. Para o autor, metacognição é "o conhecimento sobre os próprios processos cognitivos e resultados ou qualquer coisa relacionada a eles (...). O monitoramento ativo e consequente regulação e orquestração desses processos em relação aos objetos cognitivos" (FLAVELL, 1979, p. 906), ou seja, o conhecimento dos próprios processos cognitivos.

Dembo e Hillman (1976), Sternberg (2008), Matlin (2004), Fox e Riconscente (2008), Hertzog e Dunlosky (2011) e Frenkel (2014) seguem numa perspectiva teórica similar à Flavell (1979). 
Estes autores definem metacognição como o conhecimento, a consciência e o controle que a pessoa tem de seus processos cognitivos. Metacognição é a capacidade de um indivíduo para refletir e considerar cuidadosamente os seus processos de pensamentos, especialmente quanto à tentativa de reforçar as capacidades cognitivas com objetivo de regular e supervisionar a aprendizagem.

A metacognição é usada pelo estudante para reflexão sobre sua própria aprendizagem, buscando sempre novas soluções para aprender de modo a conseguir superar obstáculos. Oliveira (2009), destaca que o uso frequente das estratégias metacognitivas favorece o sucesso escolar e uma melhor apropriação do conhecimento.

\section{JOGOS DIGITAIS E EDUCAÇÃO}

Os jogos digitais conquistaram um espaço significativo na vida de crianças, jovens e adultos, e hoje é um dos setores que mais cresce na indústria de mídia e entretenimento. De acordo com dados da Pesquisa Game Brasil 2021 (SIOUX GROUP, 2021) cerca de 72\% dos brasileiros são usuários. Esses dados colaboram com o entendimento que estes artefatos fazem parte de nossa cultura e que atingem um público diversificado, de vários gêneros e classes sociais.

Na Espanha, conforme o anuário da Asociación Española de Videojuegos (AEVI, 2019), os jogos digitais apareceram como a principal escolha audiovisual e cultural, com um faturamento 1.479 milhões de euros para a indústria dos jogos. O perfil dos jogadores apresenta que 58\% dos jogadores se autodeterminam do sexo masculino e $42 \%$ do sexo feminino. De modo geral esses jogadores dedicam 6,7 horas por semana para jogar.

Nas instituições de ensino existe um crescente interesse entre pesquisadores e professores em descobrir de que formas os jogos digitais podem ser usados como recurso para apoiar a aprendizagem e quais são os seus benefícios (KIRRIEMUIR; MCFARLANE, 2004). Ter componentes de prazer e diversão inseridos nos processos de estudo é importante porque, com o aluno mais relaxado, geralmente há maior recepção e disposição para o aprendizado (PRENSKY, 2001).

Jogos bem projetados levam os jogadores para um estado de intensa concentração e envolvimento entusiasmado (chamado de estado de fluxo), onde a ânsia por vencer promove o desenvolvimento de novas habilidades (MITCHELL; SAVILL-SMITH, 2004). A tendência hoje é de que as tecnologias digitais ampliem a presença nas práticas de ensino e, nesse contexto, entende-se que os jogos digitais podem ser elementos significativos para enriquecer aulas e fortalecer as estratégias de aprendizagem. 


\section{TRABALHOS CORRELATOS}

Em trabalhos similares, em sua revisão sistemática de literatura, Clark et al. (2016) revisaram, estudaram o impacto detalhado dos jogos digitais nos resultados da aprendizagem, acreditaram que os jogos são importantes no apoio à aprendizagem produtiva e destacaram o importante papel do design de jogos.

Seguindo a mesma linha de pensamento, Backlund e Hendrix (2013) relataram em sua revisão que o uso de jogos sérios no processo educacional gerou resultados positivos na aprendizagem. Wouters (2013), realizou técnicas de meta-análise e também usa métodos comparativos para estudar se os jogos são mais eficazes e motivados do que os métodos tradicionais de ensino. Em comparação com os métodos tradicionais de ensino, eles descobriram que a aprendizagem e a retenção eram mais eficazes, mas menos motivadas.

Karam, em Rozo (2011), fez uma revisão de estudos que relacionam os videogames ao fortalecimento do pensamento criativo, observando que os jogos podem ser usados não apenas como uma ferramenta, mas também como uma estratégia geral para ativar julgamentos metacognitivos. O estudo deu ênfase aos elementos cognitivos e emocionais encontrados nos jogadores (como as habilidades de concentração), propondo o desenvolvimento centrado no jogo para fortalecer as estratégias de ativação dos julgamentos de metamemória e metacognição.

Na temática de Aprendizagem Baseadas em Jogos (GBL), Braad, Degens, Ijsselsteijn (2020) em uma revisão qualitativa discutiram os objetivos, intervenções e efeitos relatados em estudos que abordam a metacognição em GBL , com o objetivo de informar designers educacionais, pesquisadores e outros profissionais que desejam abordar a metacognição nessa abordagem de aprendizagem inovadora.

Destacando a autorregulação que é um processo ativo no qual os estudantes monitoram, regulam e controlam suas cognições, Anthonysamy; Koo e Hew, (2020) relatam em uma RSL sobre as estratégias de aprendizagem autorregulada utilizadas pelos estudantes universitários para seu aprendizado. Ao final, com 14 artigos selecionados, foi identificada uma lista de estratégias de aprendizagem autorregulada no que refere ao conhecimento metacognitivo, gestão de recursos e estratégias de crença motivacional para o desempenho da aprendizagem. Nas observações dos autores citados, os jogos costumam ser mais eficazes quando considerados como complemento de outros métodos de ensino e envolvem os alunos em discussões em grupo e várias sessões de treinamento. 


\section{PERGUNTAS DA RSL}

Em busca de respostas que possam responder se os estudantes fazem uso da metacognição quando utilizam jogos digitais em seu processo de aprendizagem, este estudo apresenta algumas questões de pesquisa:

QP1: Nos estudos desenvolvidos, foi identificado que os estudantes ao fazerem uso dos jogos digitais possuíam a consciência sobre os processos cognitivos que estavam utilizando?

QP2: Nas pesquisas realizadas, os alunos utilizaram os processos metacognitivos (conhecimento, monitoramento e o controle) nos jogos?

QP3: Os artigos selecionados indicaram se os alunos fizeram uso de habilidades e estratégias metacognitivas durante a utilização dos jogos?

\section{MÉTODO}

Esta RSL foi desenvolvida a partir dos estudos de Sampaio e Mancini (2007). Para esses autores, cinco passos devem ser seguidos, conforme ilustrado na Figura 1.

Figura 1. Etapas para elaboração da revisão

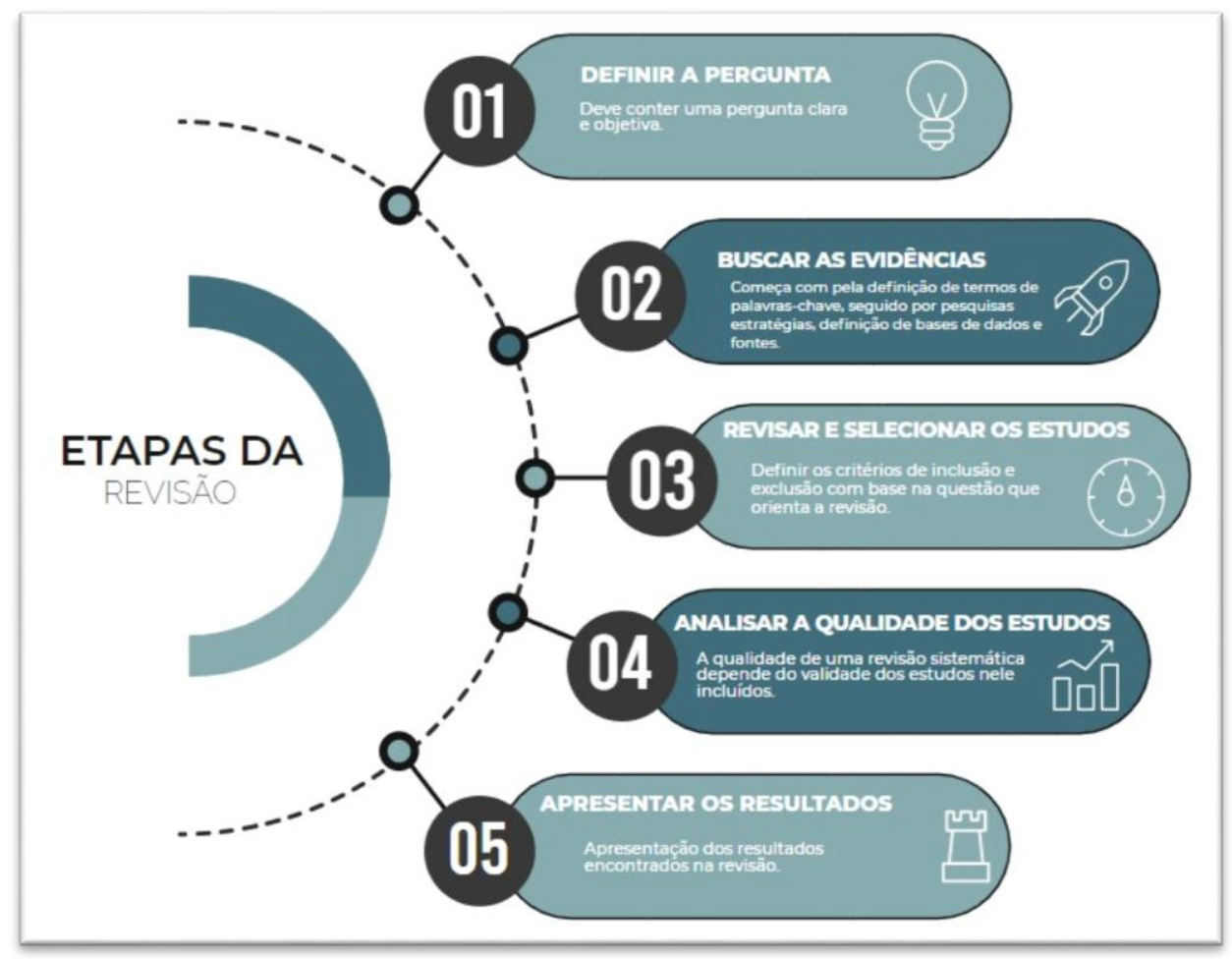

Fonte: Os autores (2021), adaptado de Sampaio e Mancini, (2007; p. 79:80). 


\section{Termos de busca}

A presente RSL, teve como objetivo identificar os estudos em que os alunos que utilizam os jogos digitais em seu aprendizado, fizeram uso da consciência, habilidades e estratégias metacognitivas. Para a busca deste objetivo, as seguintes palavras-chave foram usadas: "metacognição", "processos metacognitivos", "conhecimento metacognitivo", "monitoramento metacognitivo", "controle metacognitivo", "jogos digitais" e "habilidades e estratégias", conforme apresentadas no quadro 1, abaixo.

Quadro 1. Termos de buscas

\begin{tabular}{|l|l|l|}
\hline \multicolumn{1}{|c|}{ Termos } & \multicolumn{1}{c|}{ Sinônimos } & \multicolumn{1}{c|}{ Tradução } \\
\hline Metacognition & & Metacognição \\
\hline Metacognitive processes & & Processos metacognitivos \\
\hline Metacognitive knowledge & & Conhecimento metacognitivo \\
\hline Metacognitive monitoring & & Monitoramento metacognitivo \\
\hline Metacognitive control & & Controle metacognitivo \\
\hline Digital games & Games & Jogos digitais \\
\hline Skills and Strategies & & Habilidades e estratégias \\
\hline
\end{tabular}

Fonte: os autores (2021)

\section{String de busca.}

Foi utilizada a seguinte string de busca: (metacognition OR "metacognitive knowledge" OR "metacognitive monitoring" OR "metacognitive control" OR "metacognitive processes") AND (“digital games" OR games) AND skills AND strategies baseada no quadro 1, e para atender as necessidades das perguntas da RSL.

\section{Entendendo o que se procura}

As palavras-chaves foram definidas para uma melhor efetividade nas buscas nas bases de conhecimentos pesquisada e a procura por "Habilidades e estratégias", representam as aptidões e procedimentos que funcionam melhor para o aluno, e como e quando usá-las.

Estávamos também em busca, dos principais elementos metacognitivos, que para os autores citados metacognição engloba três itens: conhecimento, o monitoramento e o controle metacognitivo (METCALFE, 2009;DEFFENDI; BORUCHOVITCH, 2016; FLAVELL, 1979;DOYLE, 2013). 
O conhecimento metacognitivo tem a ver com a cognição propriamente dita. Segundo Deffendi e Boruchovitch (2016; p. 57) “(...) envolve o conhecimento acerca da cognição, por exemplo, sobre como ocorre a aprendizagem ou como melhorá-la, ou ainda, sobre o que o indivíduo entende o que consegue ou não fazer a partir de suas habilidades cognitivas". Boruchovitch (1999; p. 6) relata que esses processos cognitivos dizem respeito ao "1) conhecimento sobre si mesmo (pontos fortes, pontos fracos, preferências pessoais); 2) conhecimento sobre a tarefa (níveis de dificuldade, demandas); e, 3) conhecimento sobre o uso de estratégias (quais, quando, por quê e para quê)".

O monitoramento metacognitivo se refere à avaliação ou o julgamento do estado atual de uma atividade cognitiva e/ou do progresso durante a realização de uma tarefa cognitiva. Como exemplos de ações da ordem do monitoramento cognitivo, pode-se citar: a auto avaliação da adequação das estratégias usadas para solucionar um problema e o autoexame acerca do quão bem está se compreendendo o que se estuda (DEFFENDI; BORUCHOVITCH, 2016).

O controle metacognitivo diz respeito à regulação da atividade cognitiva em curso; refere-se às ações ou providências que devem ser tomadas a partir das informações provenientes do monitoramento cognitivo, tendo em vista regular ou ajustar aspectos de uma tarefa cognitiva. Neste estágio, Boruchovitch (2008) fala da necessidade do indivíduo ter plena consciência dos objetivos que deseja alcançar; conhecer as demandas e as exigências da tarefa; procurar realizar as ações; avaliar o nível de realização atingido e, num processo cíclico, alterar, se necessário, os procedimentos adotados quando não alcançar resultados esperados.

Os processos cognitivos dizem respeito a utilização da consciência metacognitiva a qual é estimulada desde quando os jogadores se preparam para jogar, segundo (DOOLITTLE, 1995), mesmo em jogos mais simples podem provocar a prática de metacognição e promover a consciência metacognitiva.

As habilidades metacognitivas dizem respeito ao ganho de experiências com os jogos digitais em busca de seu aprendizado para incorporá-lo as suas atuais estruturas de conhecimento sobre um determinado domínio (CHRISTOPH, 2006). Devem conjeturar sobre o que estão fazendo e sobre o processo de aprendizagem diante da situação proposta, principalmente para obter uma visão sobre o que deu certo ou errado e buscar os caminhos e as soluções específicas para fazer frente aos desafios encontrados. Em um conceito muito próximo, as estratégias metacognitivas, que segundo (SHANNON, 2008) são quando refletem sobre quais táticas se adaptam melhor ao seu estilo de aprendizagem.

No contexto educacional, os elementos procurados podem fornecer elementos bases na 
definição de diretrizes curriculares, assim como no desenvolvimento de estratégias de ensino e aprendizagem.

\section{Base de dados pesquisadas}

As fontes deveriam estar disponíveis via web, preferencialmente em bases de dados científicas da área. Este processo será realizado por meio de buscas formadas por palavras-chave. Os trabalhos foram encontrados a partir de pesquisas realizadas em portais de busca de artigos, teses, dissertações e periódicos. Durante o procedimento de recuperação das informações foram consideradas as strings encontradas preferencialmente em Títulos, Resumos e Palavras-chave de cada base de dados.

As bases de dados utilizadas nesta pesquisa foram: $\mathrm{Scielo}^{3}, \mathrm{Scopus}^{4}, \mathrm{Springer}^{5}, \mathrm{IEEE}^{6}, \mathrm{ACM}^{7}$, Pubmed $^{8}$, APA Psycnet ${ }^{9}$, BVS Psico BR ${ }^{10}$, ERIC $^{11}$, Research Gate ${ }^{12}$, Wiley ${ }^{13}$ e ScienceDirect ${ }^{14}$.

\section{Critérios de Inclusão e Exclusão}

Para pesquisas obtidas por meio de buscas efetuadas nas bases de dados, se faz necessário adotar alguns critérios de inclusão da pesquisa para selecionar claramente os trabalhos que fazem uso da metacognição em jogos digitais. Para o propósito desta RSL, os critérios de inclusão, foram: os trabalhos que utilizem a metacognição nos jogos digitais; publicados e disponíveis integralmente nas bases científicas buscadas; que façam uso do conhecimento, controle ou monitoramento cognitivo e que utilizem as habilidades metacognitivas nos jogos digitais.

Os de exclusão, foram assim definidos: os trabalhos que não façam uso dos processos cognitivos; publicados antes de 2010; que não sejam no ensino superior, e que palavras de busca não apareçam no título, resumo e nas palavras chaves do artigo.

\footnotetext{
3 http://scielo.org

4 https://www.elsevier.com/pt-br/solutions/scopus

5 https://link.springer.com/

6 https://ieeexplore.ieee.org/Xplore/home.jsp

7 https://dl.acm.org/

8 https://www-ncbi-nlm-nih.ez9.periodicos.capes.gov.br/pmc/

9 https://psycnet.apa.org

10 http://www.psi.bvs.br/

$11 \mathrm{https} / / /$ eric.ed.gov/

12 https://www.researchgate.net/

13 https://onlinelibrary.wiley.com/

14 https://www.sciencedirect.com/
} 


\section{Critérios de qualidade}

Após a seleção dos estudos pelos seus critérios descritos na etapa anterior, adotamos também critérios de seleção por qualidade de conteúdo e significância dos objetivos buscados. Foram reanalisados os estudos selecionados, observando os itens: coerência e coesão do texto; forma objetiva de uso da metacognição; se houve ou não citação aos elementos da metacognição; se fez uso para o ensino no jogo; se houve coleta e análise de dado; se utilizou algum programa para tratamento dos dados e se fez uso no ensino básico, fundamental ou superior.

\section{Ferramenta utilizada.}

Para realização deste trabalho, fizemos uso do software, StArt - (State of the Art through Systematic Review - Estado da Arte por Revisão Sistemática), que foi desenvolvida pela Universidade Federal de São Carlos (UFSCar) no Laboratório de Pesquisa em Engenharia de Software (LaPES).

O software possibilitou uma sistematização no processo de revisão de literatura, desde a inclusão das referências nas bases, com um planejamento, execução e sumarização dos resultados. No processo de execução, o Start permitiu a listagem e controle dos estudos coletados, bem como sua seleção e extração baseadas nos critérios de inclusão e exclusão. Em sumarização, são admitidas observações das etapas por estruturas de gráficos, grafos e árvores.

\section{Processo de Seleção das Publicações}

Inicialmente foram executadas as Strings de busca adaptadas em cada uma das bases de dados. A partir da lista de estudos retornados, foram exportados os resultados em formato BIBtex, para importação na ferramenta Start. 770 estudos foram selecionados (Figura 2).

Na etapa de seleção, foi executada a análise de cada título de cada um dos estudos, descartando aqueles que claramente não estejam relacionados à estratégia de busca, ou que não preencham os critérios de Inclusão, Critérios de qualidade ou estejam relacionados aos critérios de Exclusão definidos. Estudos excluídos nesta fase são armazenados e não passaram as fases seguintes. Nesta etapa, 58 estudos corresponderam aos critérios de inclusão.

A lista de trabalhos selecionados foi submetida a etapa de Extração. Neste segundo passo, foram analisados o resumo e as conclusões de cada estudo. A partir desta leitura, foram avaliados os critérios de Inclusão, Exclusão e Qualidade novamente. Como resultado, a lista completa dos estudos foi de 6 trabalhos. 
Nesta etapa realizamos a leitura por completo dos estudos alocados, avaliando a qualidade e extraindo os dados básicos de caracterização do estudo, bem como os dados específicos relacionados às perguntas de pesquisa, atualizando campos de comentário e anexando os arquivos completos do trabalho.

Após finalizada a extração dos dados, os passos seguintes corresponderam à análise, interpretação e documentação dos resultados, com a redação de artigo de revisão com apresentação dos resultados, métodos e técnicas adotadas na RSL.

Figura 2. Procedimentos na análise de dados.

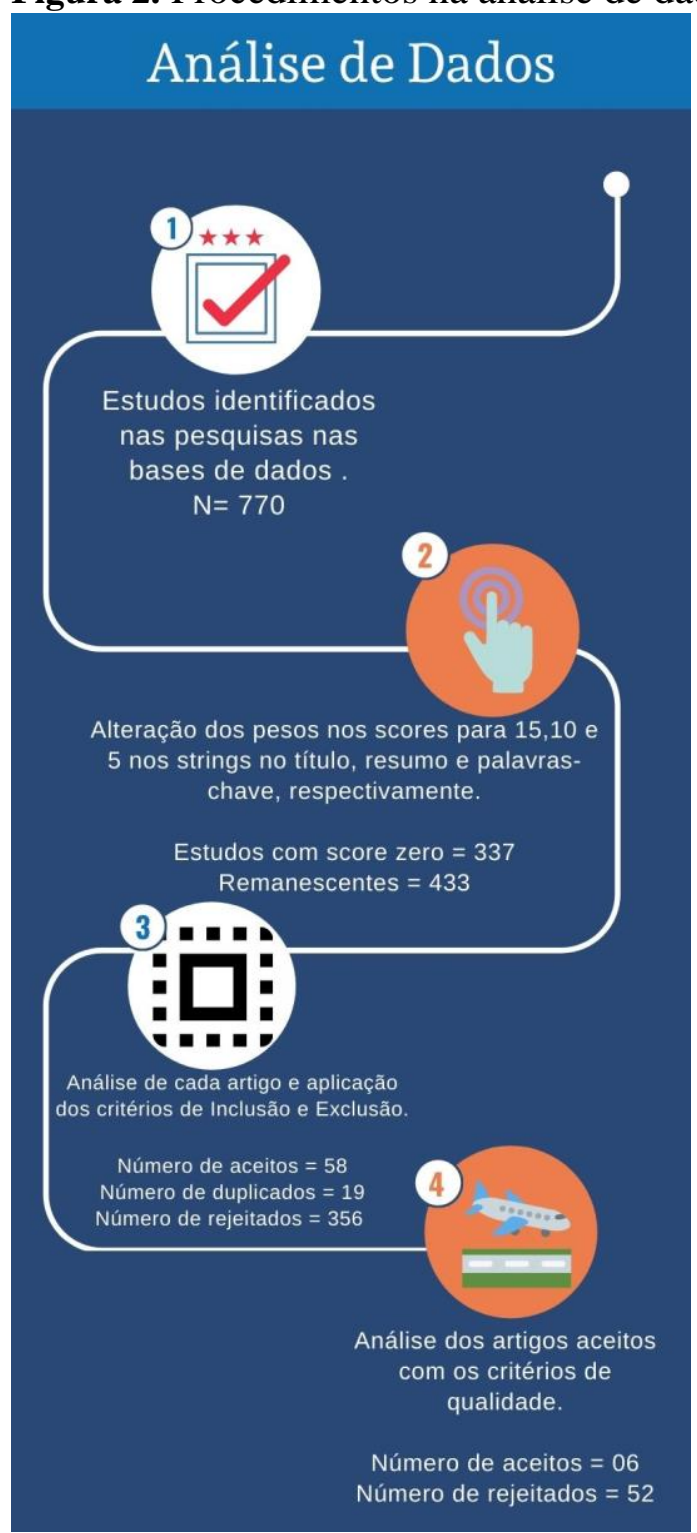

Fonte: Os autores (2020) 


\section{Utilização dos Scores dos estudos.}

Após a execução da string de buscas nas bases de dados, como já descrito, obtemos 770 estudos que possuíam as palavras-chaves no título, resumo, palavras-chaves e conteúdo, conforme mostra o Gráfico 1.

Gráfico 1. Resultados das buscas nas bases de dados

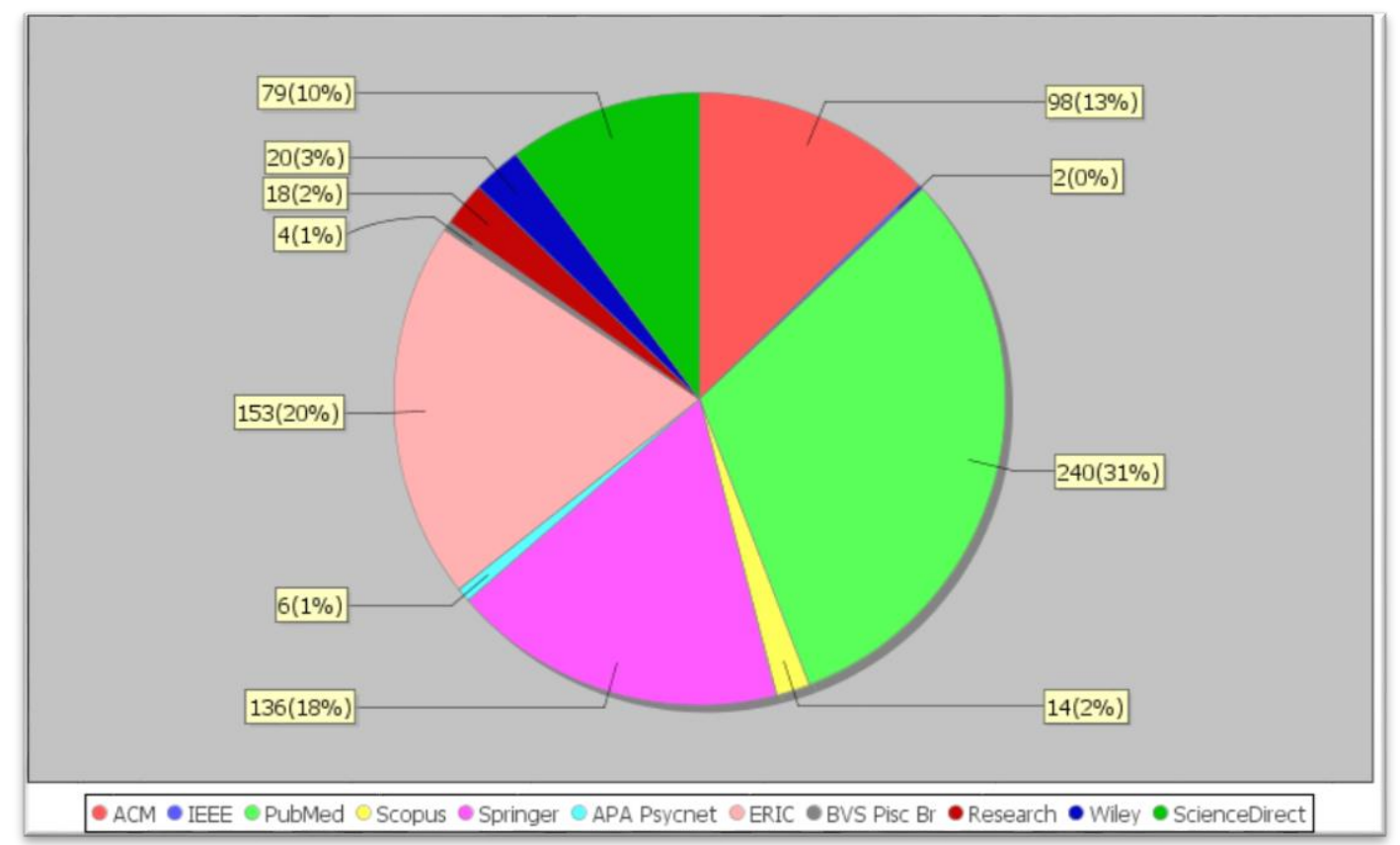

Fonte: Dados da pesquisa (2020)

Como o resultado foi expressivo e inesperado, resolvemos adotar o ajuste de pontuação para o score de cada estudo, fazendo as alterações de localização da palavras-chaves do título, resumo e palavras-chaves, como os respectivos valores: 15,10 e 5 .

Essa facilidade permitiu uma melhor visualização, tendo em vista que a busca também acontece no conteúdo do estudo e pelo simples fato de utilização daquela palavra, satisfez a condição de busca da string utilizada, no entanto, o estudo, não é objeto de nossa RSL. Na figura 3, apresentamos a tela de alteração e recalculo dos scores. 
Figura 3. Tela de alteração dos componentes dos Scores dos estudos

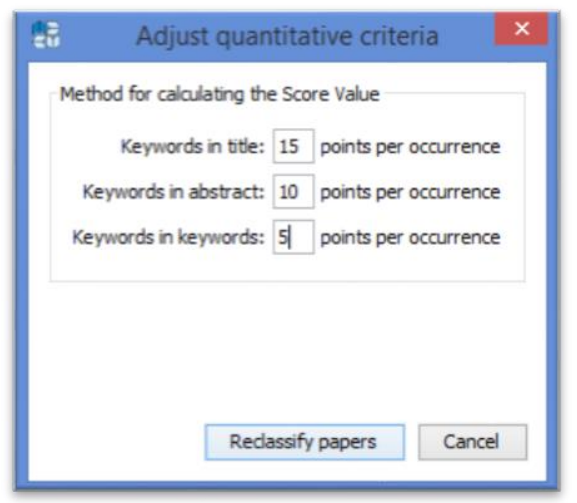

Fonte: Opção de ajustes, na ferramenta Start, no menu Review (2021)

Após clicar na opção "Reclassify papers", a coluna score dos estudos ganharam novos valores (Figura - 4), evidenciando e aumentando o peso por estarem nos lugares objetos de estudo e pesquisa principal. Dos 770 estudos iniciais, 337 apresentaram score zero, reduzindo a base de resultados para 433.

Figura 4. Tela dos estudos com scores em ordem decrescente

\begin{tabular}{|c|c|c|}
\hline Title & Year & Score \\
\hline Digital Games, Design, and Learning: A Systematic Review and Meta-Analysis.Knowing Ourselves Together: The... & & 565 \\
\hline Digital Games, Design, and Learning: A Systematic Review and Meta-Analysis.Knowing Ourselves Together: The... & & 5650 \\
\hline Hypothetico-deductive thinking as a metacognitive knowledge acquisition strategy in inquiry-based learning envi... & 2001 & 160 \\
\hline Schoolchildrenâ€"'s Compensatory Strategies and Skills in Relation to Attention and Executive Function App ... & 2019 & 130 \\
\hline Player transfer: How learning transfer and serious games answer serious (and transferable) questions about on... & 2008 & 115 \\
\hline Video games: A significant cognitive artifact of contemporary youth culture & 2005 & 115 \\
\hline 21st Century Skills: Student Perception of Online Instructor Role & 2018 & 110 \\
\hline Developing Elementary School Students' Mental Computation Skills through Didactic Games & 2020 & 110 \\
\hline Effect of the Childrenâ€'N"s Health Activity Motor Program on Motor Skills and Self-Regulation in Head Start Pr... & 2016 & 105 \\
\hline Language Games in Teaching and Learning English Grammar: A Literature Review & & 105 \\
\hline Self-directed learning in video games, affordances and pedagogical implications for teaching and learning & 2020 & 105 \\
\hline A multidimensional repertory grid as a graphic organizer for implementing digital games to promote studentsâ€ $€^{\mathrm{TM}} \ldots$ & 2021 & 100 \\
\hline Digital gaming and metacognition in middle childhood & 2021 & 100 \\
\hline EFL Teachers' Perceptions: Challenges and Coping Strategies of Integrated Skills Approach (ISA) Implementatio... & 2020 & 100 \\
\hline
\end{tabular}

Fonte: Tela de estudos selecionados do StArt (2021)

Fazendo uso dos critérios de inclusão e exclusão, apenas 58 estudos foram aceitos. A grande maioria, não faziam uso da metacognição ou de jogos digitais. Na etapa de análise dos critérios da qualidade, apenas 6 estudos foram selecionados, satisfazendo a utilização dos componentes metacognitivo no ensino superior. Na figura 5, são mostradas as associações dos estudos e seus critérios de qualidade assinalados. 
Figura 5. Grafo associando os estudos aceitos e seus critérios de qualidade

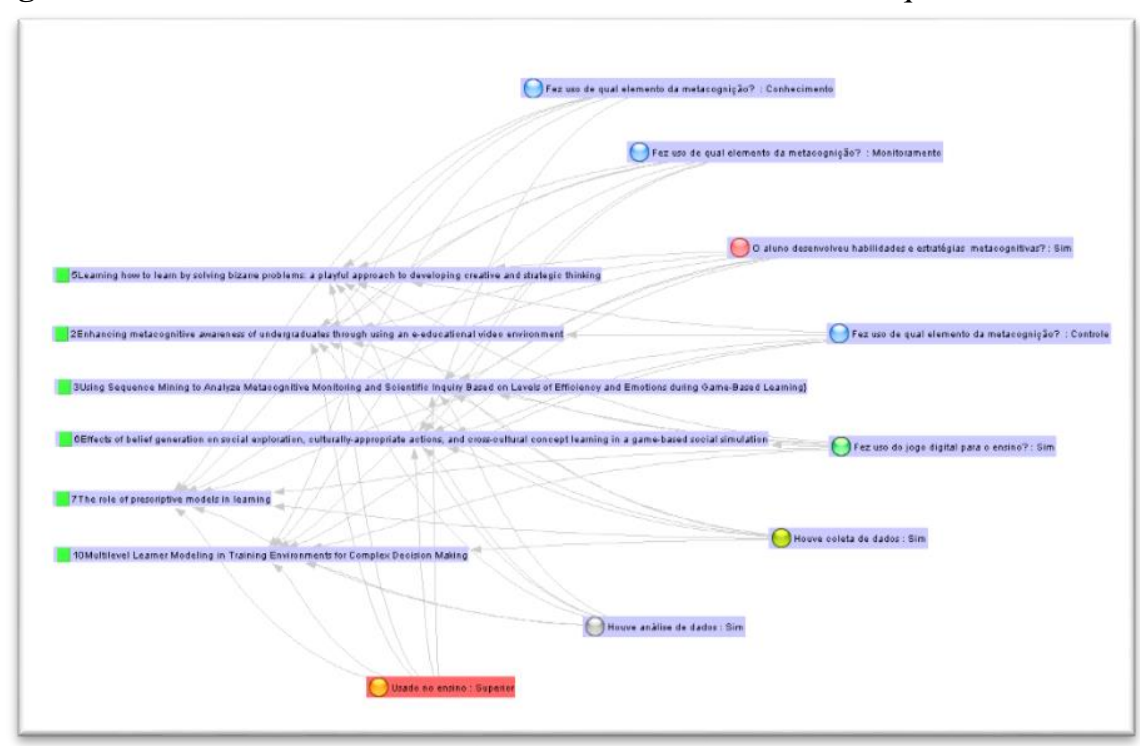

Fonte: Dados da pesquisa (2021)

A opção de sumarização da ferramenta StArt, permite a visualização de várias estruturas gráficas para todas as etapas da revisão e pelos critérios: aceitos, rejeitados, duplicados e ainda não classificados.

Figura 6. Grafo com associação das referências entre os estudos selecionados

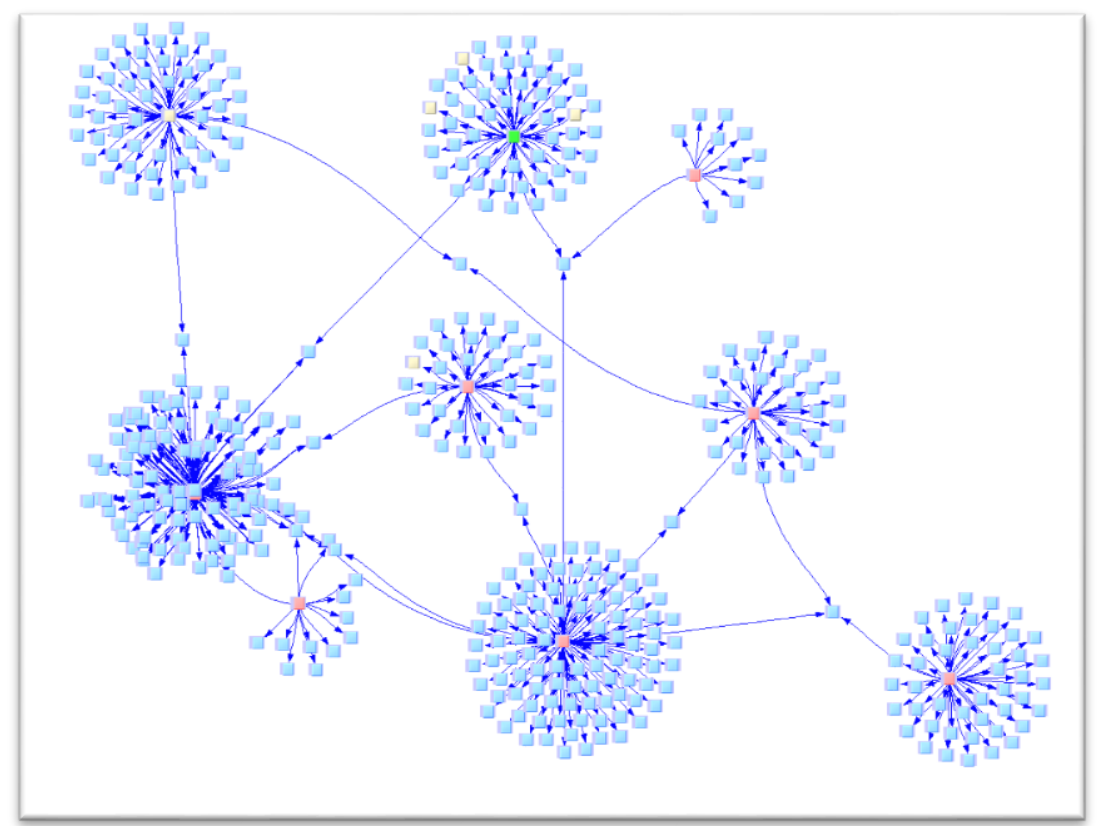

Fonte: Dados da pesquisa (2021) 
No grafo da Figura - 6, acima, uma associação das referências dos estudos selecionados. Possibilita a leitura e observação dos estudos similares, basta um duplo click em um dos ícones para abrir os dados básicos do estudo selecionado.

\section{ANALISE DOS DADOS}

Nessa etapa, com os artigos selecionados, iniciamos as respostas das perguntas. Para esse feito, fizemos uso do Microsoft Excel para tabulação dos itens: QP1(Sim, Não); QP2 (Conhecimento, Monitoramento, Controle); QP3 (Habilidades, Estratégias).

Para cada um dos seis artigos, foram observados e colocados em colunas, após, totalizados e calculados relativamente suas observações e transformados em gráficos para as questões 2 e 3 .

Dos seis artigos, em quatro deles (ALTIOK; BAŞER; YÜKSELTÜRK, 2019), (MARONE; STAPLES; GREENBERG, 2016), (GJICALI; FINN; HEBERT, 2020), (BISWAS et al., 2019), fizeram uso explícito dos processos cognitivos, mostraram o conhecimento sobre a tarefa à qual estavam realizando, tanto sobre si mesmo, ou sobre o uso das estratégias utilizadas nos experimentos propostos. Em Taub e Azevedo (2018) foi efetivado um estudo dirigido para a autorregularem do aprendizado conduzida pelo monitoramento metacognitivo, com foco nas emoções e motivação para os jogos digitais. Em Sandberg; Wielinga e Christoph (2012) a pesquisa foi sobre o jogo $\mathrm{Km}$ Quest ${ }^{15}$ com objetivo de apoiar os alunos na aquisição dos conhecimentos declarativos e procedimentais.

O resultado da QP2, pode ser visto na Figura 7. O conhecimento metacognitivo, se fez presente em $63 \%$ dos artigos selecionados, mostrando que os alunos entendem o que devem fazer com suas habilidades cognitivas em seu aprendizado quando estão fazendo uso dos jogos digitais.

Figura 7. Resultado da QP2

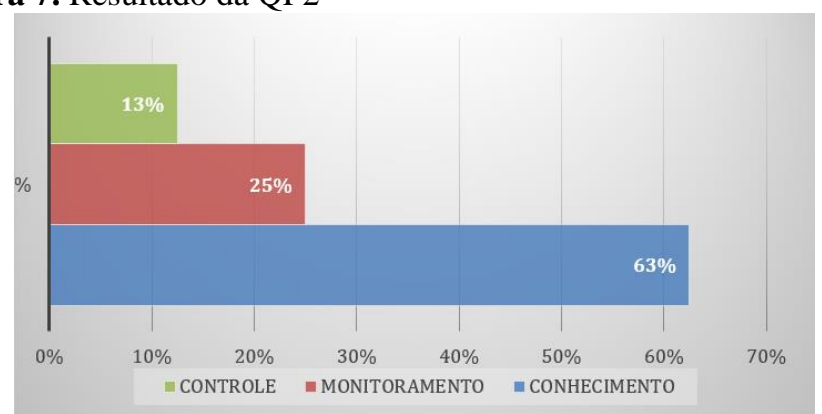

Fonte: Os autores (2021).

\footnotetext{
${ }^{15}$ Um jogo de simulação colaborativo baseado na Internet para aprender a resolver problemas de gestão do conhecimento.
} 
O monitoramento metacognitivo, apresentou que $25 \%$ deles, verificam como estão em seu desempenho nos jogos e fazem uma auto avaliação das estratégias utilizadas para resolver os problemas existentes e procuram os melhores procedimentos para realização dos desafios propostos; No entanto, quanto as ações ou providências que devem ser tomadas a partir do monitoramento para regular ou mesmo ajustar algum procedimento, que denominamos de controle metacognitivo, esse percentual se apresenta como o menor deles, apenas 13\%, possuem a plena consciência dos objetivos que desejam alcançar.

Figura 8. Resultado da QP3.

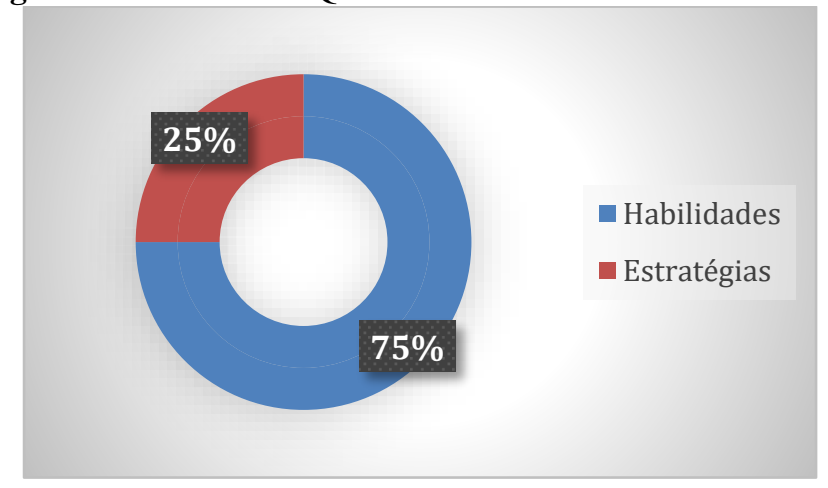

Fonte: Os autores (2021).

Na QP3, estávamos em busca das habilidades e estratégias dos processos que permitem monitorar, regular e controlar a cognição com a utilização dos jogos digitais. A maioria faz uso das habilidades metacognitiva (75\%), buscam com elas caminhos e soluções específicas diante dos desafios propostos pelos jogos. No entanto, fazem pouco uso das estratégias (25\%) que permitiriam refletir sobre as táticas ou procedimentos a serem adotados para superar os desafios propostos (Figura 8).

\section{CONCLUSÕES}

A mudança nos métodos de ensino de um ambiente centrado na sala de aula para um ambiente centrado no aluno, e o uso crescente de jogos como uma tecnologia de aprendizagem inovadora, exigem mudanças no processo de ensino (VLACHOPOULOS; MAKRI, 2017), a este respeito, espera-se que os jogos digitais desempenhem um papel significativo no processo de aprendizagem.

A presente revisão, manteve seu foco na utilização dos elementos que configuram a utilização da metacognição, ou seja, a consciência de utilização dos processos cognitivos; as habilidades e estratégias e principalmente, os três elementos principais que indicam a utilização da metacognição: conhecimento, monitoramento e controle metacognitivo, e sua relação com os 


\section{Revista Docência e Cibercultura}

jogos digitais.

Após análise de todos os estudos filtrados nas bases de conhecimento, observamos que por ser um campo de estudo novo, com poucos trabalhos científicos direcionados para a totalidade dos elementos metacognitivos, concluímos, com base na amostra selecionada, na aceitação da hipótese de que os alunos que utilizam os jogos digitais em seu aprendizado, fazem uso da consciência, habilidades e estratégias metacognitivas.

\section{REFERÊNCIAS}

ALTIOK, S.; BAŞER, Z.; YÜKSELTÜRK, E. Enhancing metacognitive awareness of undergraduates through using an e-educational video environment. Computers and Education, v. 139, p. 129-145, 2019.

ANTHONYSAMY, L.; KOO, A. C.; HEW, S. H. Self-regulated learning strategies and nonacademic outcomes in higher education blended learning environments: A one decade review. Education and Information Technologies, n. September, 2020.

BISWAS, G. et al. Multilevel Learner Modeling in Training Environments for Complex Decision MakingIEEE Transactions on Learning Technologies, 2019.

BORUCHOVITCH, E. Estratégias de aprendizagem e desempenho escolar: considerações para a prática educacional. Psicologia: Reflexão e Crítica, v. 12, n. 2, p. 361-376, 1999.

BRAAD, E.; DEGENS, N.; IJSSELSTEIJN, W. A. Designing for metacognition in gamebased learning: A qualitative review. Translational issues in psychological science, v. 6, n. 1, p. 53, 2020.

CHRISTOPH, L. H. The role of metacognitive skills in learning to solve problems. [s.l: s.n.].

CLARK, D. B.; TANNER-SMITH, E. E.; KILLINGSWORTH, S. S. Digital Games, Design, and Learning: A Systematic Review and Meta-Analysis. Review of Educational Research, v. 86, n. 1, p. 79-122, 2016.

DEFFENDI, L. T.; BORUCHOVITCH, E. Avaliação do monitoramento metacognitivo: análise da produção científica. Avaliação Psicológica, 2016, 15(n. esp), p. 57-65, 2016.

DEMBO, H. M. Learning To Teach Is Not Enough — Future Teachers Also Need To Learn How To Learn. Teacher Education Quarterly, v. 28, 2001.

DEMBO, M. H.; HILLMAN, S. B. An instructional model approach to educational 
psychology. Contemporary Educational Psychology, v. 1, n. 2, p. 116-123, 1976.

DOOLITTLE, J. H. Using Riddles and Interactive Computer Games to Teach ProblemSolving Skills. Teaching of Psychology, v. 22, n. 1, p. 33-36, 1995.

DOYLE, B. Metacognitive Awareness: Impact of a Metacognitive Intervention in a Prenursing Course. [s.1.] LSU Doctoral Dissertations, 2013.

EGENFELDT-NIELSEN, S. The challenges to diffusion of educational computer games. Leading Issues in Games Based Learning, v. 141, 2010.

FLAVELL, J. H. Metacognition and Cognitive Monitoring. American Psychological Association, v. 34, n. 10, p. 906-911, 1979.

FOX, E.; RICONSCENTE, M. Metacognition and self-regulation in James, Piaget, and Vygotsky. Educational Psychology Review, v. 20, n. 4, p. 373-389, 2008.

FRENKEL, S. Metacognitive components in learning to learn approaches. International Journal of Psychology: a Biopsychosocial Approach, v. 14, n. December 2014, p. 95-112, 2014.

FUND, E. E. Metacognition and Self-regulated Learning: Guidance Report. The Sutton Trust-Education Endowment Foundation Teaching and Learning Toolkit, p. 1-30, 2018.

GJICALI, K.; FINN, B. M.; HEBERT, D. Effects of belief generation on social exploration, culturally-appropriate actions, and cross-cultural concept learning in a game-based social simulation. Computers and Education, v. 156, n. March, p. 103959, 2020.

GROS, B. The impact of digital games in education. First Monday, v. 8, n. 7, jul. 2003, v. 8, n. 7, 2003.

HENDRIX, M.; BACKLUNG, P. Educational Games - Are They Worth The Effort? Games and Virtual Worlds for Serious Applications (VS-GAMES), n. December, p. 1-8, 2013.

HERTZOG, C.; DUNLOSKY, J. Metacognition in later adulthood: Spared monitoring can benefit older adults' self-regulation. Current Directions in Psychological Science, v. 20, n. 3, p. 167-173, 2011.

KIRRIEMUIR, J.; MCFARLANE, A. Literature Review in Games and Learning. A NESTA Futurelab Research report - report 8. 2004., p. 1-40, 2004.

MARONE, V.; STAPLES, C.; GREENBERG, K. H. Learning how to learn by solving bizarre problems: a playful approach to developing creative and strategic thinking. On the Horizon, v. 24, n. 1, p. 112-120, 2016. 
MATLIN, W. M. Psicologia Cognitiva. 5a. ed. [s.1.] LTC Editora, 2004.

METCALFE, J. Metacognitive Judgments and Control of Study. Current Directions in Psychological Science- Columbia University, v. 18, n. 3, p. 159-163, 2009.

MITCHELL, A.; SAVILL-SMITH, C. The use of computer and video games for learning A review of the literature The use of computer and video games for learning. [s.l: s.n.].

OLIVEIRA, K. L. DE; BORUCHOVITCH, E.; SANTOS, A. A. A. DOS. Estratégias de aprendizagem e desempenho acadêmico: evidências de validade. Psicologia: Teoria e Pesquisa, v. 25, n. 4, p. 531-536, 2009.

PRENSKY, M. The Games Generations: How Learners Have Changed. Computers in Entertainment, v. 1, n. 1, p. 1-26, 2001.

PRENSKY, M. Digital Game-based Learning Prensky. ACM Computer in Entertainment, v. 1, n. 1, p. 1-4, 2003a.

PRENSKY, M. Digital game-based learning. Computers in Entertainment (CIE), v. 1, n. 1, p. 21, 2003b.

ROZO, J. M. K. Creatividad y videojuegos: nuevos paradigmas en la generación de aprendizaje autónomo. Revista Repertorio de Medicina y cirugia, v. 20, n. 1, p. 45-50, 2011.

SAMPAIO, R. F.; MANCINI, M. C. Systematic Review Studies: a Guide for Careful Synthesis of Scientific Evidence. Brazilian Journal of Physical Therapy, v. 11, n. 1, p. 7782, 2007.

SANDBERG, J. A. C.; WIELINGA, B. J.; CHRISTOPH, L. H. The role of prescriptive models in learning. Computers and Education, v. 59, n. 2, p. 839-854, 2012.

SAVI, R.; ULBRICHT, V. R. Jogos Digitais Educacionais: Benefícios E Desafios. Renote, v. 6, n. 1, 2008.

SHANNON, S. V. Using Metacognitive Strategies and Learning Styles to Create SelfDirected Learners. Institute for Learning Styles Journal, v. 1, n. 2001, p. 14-28, 2008. SIOUX GROUP, \& G. G. Pesquisa Game Brasil 2021. [s.l: s.n.].

STERnBERG, R. Psicologia Cognitiva. Porto Alegre: Artmed, Porto Alegre, 2008.

TAUB, M.; AZEVEDO, R. Using Sequence Mining to Analyze Metacognitive Monitoring and Scientific Inquiry Based on Levels of Efficiency and Emotions during Game-Based Learning. Journal of Educational Data Mining, v. 10, n. 3, p. 1-26, 2018. 


\section{Revista Docência e Cibercultura}

VAN ECK, R. Digital game-based learning: Still restless, after all these years. Educause Review, v. 50, n. 6, p. 13, 2015.

VAN VELZEN, J. Metacognitive learning: Advancing learning by developing general knowledge of the learning process. Metacognitive Learning: Advancing Learning by Developing General Knowledge of the Learning Process, p. 1-162, 2015.

VLACHOPOULOS, D.; MAKRI, A. The effect of games and simulations on higher education: a systematic literature review. [s.l.] International Journal of Educational Technology in Higher Education, 2017. v. 14

WOUTERS, P.; VAN OOSTENDORP, H. A meta-analytic review of the role of instructional support in game-based learning. Computers and Education, v. 60, n. 1, p. 412-425, 2013.

ZIMMERMAN, B. J. Investigating Self-Regulation and Motivation: Historical Background, Methodological Developments, and Future Prospects. American Educational Research Journal, v. 45, n. 1, p. 166-183, 2008.

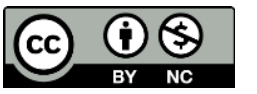

Este é um artigo de acesso aberto distribuído sob os termos da Licença Creative Commons Atribuição Não Comercial-Compartilha Igual (CC BY-NC- 4.0), que permite uso, distribuição e reprodução para fins não comerciais, com a citação dos autores e da fonte original e sob a mesma licença. 